\title{
Finite Element Analysis of Titanium-Reinforced Polyetheretherketone Fracture Plating in Long Bones*
}

\author{
Jason SANDERSON**, Nobuki MURAYAMA** and Yoshitaka NAKANISHI** \\ ${ }^{* *}$ Graduate School of Science and Technology, Kumamoto University \\ 2-39-1 Kurokami, Kumamoto, Japan \\ E-mail: jason.m.sanderson@gmail.com
}

\begin{abstract}
The present study uses finite element analysis (FEA) to compare an original long bone plate design consisting of a titanium alloy reinforced polyetheretherketone (PEEK) biomaterial with fracture plates made from a standard titanium alloy. The original plate design consists of carbon reinforced PEEK with several independent, cylindrical, titanium reinforcements spanning the length and width of the plate. Standard plates are equivalent in dimension to reinforced PEEK plates but are made of solid titanium alloy. An anatomically correct human tibia model is used to create two bone fragments, proximal and distal, simulating a simple transverse fracture. The construct is loaded proximally upon the medial and lateral tibia plateaus and fixed distally at the talocrural joint surface. Screw-plate contact conditions are defined as "bonded" to simulate locking screws, and screw-bone contacts are "separation-no sliding" to simulate the ability of the screw to pull away from but not slide along the bone-screw interface. The FEM analysis showed that the reinforced PEEK implants are successful in reducing stress shielding and average screw stress across all constructs when compared to standard Ti-alloy plates. The reinforced plates also allow micro-motion, which is conducive to secondary healing, whereas the standard plates inhibit this type of motion.
\end{abstract}

Key words: Fracture Plating, PEEK, Finite Element Analysis, Fracture Fixation, Orthopedics, Biomaterials

\section{Introduction}

Surgeons have several options to choose from to stabilize tibia fractures and expedite the fracture healing process. The least invasive and most often used option is plaster or fiberglass cast immobilization. Bone fractures will usually heal on their own if the bone fragments are properly aligned and stabilized. If a fracture is severe enough, external fixation may be used. In these cases, metal pins are inserted through the soft tissue and into the bone on both the proximal and distal ends of the break. This method stabilizes the fracture with a structure on the outside of the body and is often used when the tissue around the fracture requires further healing before surgery can be performed. If a fracture is too severe for casting but the soft tissue damage is limited, open reduction and internal fixation is recommended. This technique allows a surgeon to return the bones to proper position and stabilize the bone fragments by using metal screws between the bone fragments, a metal plate that spans across the fracture, or an intramedullary rod through the center of the bone. These techniques are extremely successful but are still not without problems.

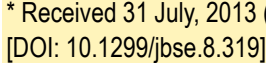

Copyright (c) 2013 by JSME 
An issue often reported with repairing bone fractures with metallic implants is stress shielding ${ }^{(1,2)}$. This happens when stiff metallic implants insulate the bone from incurred stress, often at the fracture site, thereby causing it to weaken. This weakening can lead to delayed union, refracture, or implant failure ${ }^{(3,4)}$. To reduce stress shielding caused by orthopedic implants, it has been suggested that the construct should have a Young's modulus closer to that of bone ${ }^{(5,6)}$. In a previous study, we analyzed fracture plates made from natural polyetheretherketone (PEEK) and carbon reinforced PEEK both experimentally and with FEA. It was found that plates made of only PEEK materials were likely too soft to be used in long bone fractures ${ }^{(7)}$. We suggested adding an inner reinforcement to the PEEK plates to add strength to the construct. The present study presents a new orthopedic plate design made of PEEK with a titanium-alloy inner support and compares it to a titanium-alloy plate of same dimension using finite element analysis.

\section{Materials and Methods}

\subsection{Fracture Plate and Screw Models}

Two types of custom fracture plates were designed in Autodesk Inventor Professional 2013®, a standard plate (SPlate) and a titanium reinforced polyetheretherketone (PEEK) plate (RPlate). Plate dimensions were $200 \times 20$ × $5 \mathrm{~mm}$, and contained 14 tapered, lockingscrew holes. The SPlate was solid titanium and the RPlate was made from carbon reinforced (30\%) PEEK with 13 cylindrical inner titanium reinforcements crossing the plate between each screw hole and 2 long inner reinforcements running the length of the plate (Fig. $1 \mathrm{a}, \mathrm{b}$ ).

\section{Locking screws were designed with tapered screw heads to match plate holes, and simple cylindrical shafts. Screw threads were not included due to the significant increase

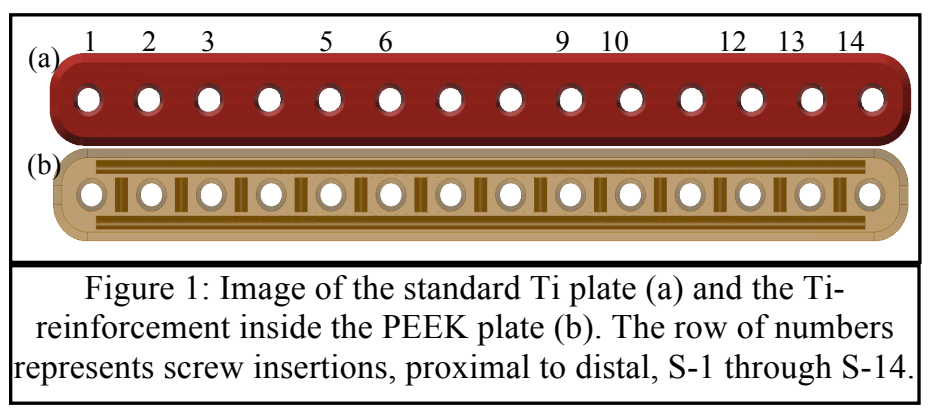 computational time they would generate versus the assumed minor effect they would have on this type of analysis ${ }^{(4)}$.}

\subsection{Tibia Bone Model}

An anatomically correct CAD model of the left tibia was obtained from the VAKHUM (Virtual Animation of the Kinematics of the Human) public dataset ${ }^{(8)}$. A medullary canal was created through the center of the diaphysis extending from the distal end of the tibial

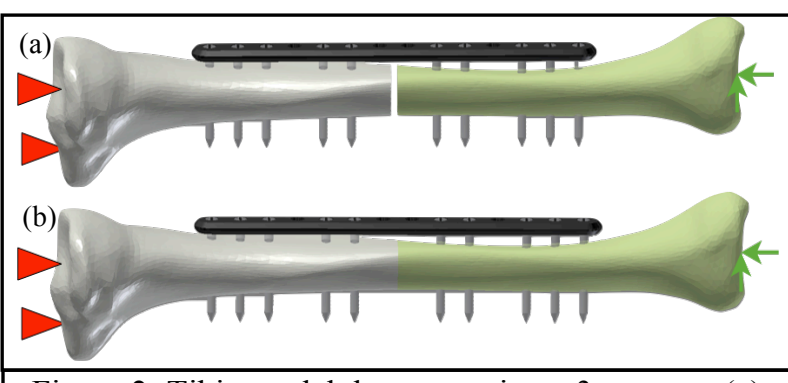

Figure 2: Tibia model demonstrating a $3 \mathrm{~mm}$ gap (a) and non-gap (b). Approximate position of the FEA load (150 N medially, $60 \mathrm{~N}$ laterally) is displayed by red arrows (proximal) and the approximate area of fixation is shown by the green arrows (distal). tuberosity to approximately 6 $\mathrm{cm}$ above the medial malleolus creating an average cortical bone thickness of $5 \mathrm{~mm}$. Five screw holes per bone fragment were created for construct fixation. Bone is considered an orthotropic material, however, Peng et al. ${ }^{(9)}$ determined that under certain loading conditions, axial with a slight moment of rotation, results obtained from analysis of 
systems containing orthotropic and isotropic properties of bone are nearly identical. Therefore, density related, isotropic material properties for normal healthy bone were used to define the tibia model. This analysis is intended to approximate the initial stages of fracture healing before a fracture callus forms or direct healing begins, therefore no fracture callus is modeled. Loading is intended to simulate passive exercise as performed during physical therapy or very light loading that may occur while the patient is on crutches. A simple fracture was simulated with a transverse osteotomy through the center of the diaphysis. Two types of fracture gaps were simulated, a $3 \mathrm{~mm}$ gap construct and a non-gap construct (Fig. 2 a, b). The proximal and distal osteotomy surfaces were assigned "separation" contact type to prevent penetration between the bone fragments during simulation.

\subsection{FEA Configuration}

Normally, the tibia endures a total load of approximately 0.46 times body weight (BW) while standing ${ }^{(10)}$. To simulate load after surgery, $30 \%$ of an estimated average weight of $700 \mathrm{~N}$ (total $210 \mathrm{~N}$ ) was employed, and to produce a slight moment of rotation, loading of $150 \mathrm{~N}$ on the medial tibial plateau and $60 \mathrm{~N}$ on the lateral tibial plateau was applied. Construct movement was restrained distally at the tibial surface of the talocrural joint, and contacts between the parts of the construct were locally meshed at $1 \mathrm{~mm}$. The 14-hole plate was attached to the bone with locking screws as described by the numbers in figure 1 . To simulate locking screw connections the screw heads were assigned "bonded" contacts with plate screw holes. Screws were assumed to be able to pull away from the surface of the bone screw holes but not slide out thus "separation/no sliding" contacts were used to simulate the attachment of the screws to the bone. The working length (i.e., the distance between the two screws closest to the fracture gap) of the plate was approximately $35 \mathrm{~mm}$ (Fig. 2). Material properties for cortical bone were collected from the literature and assumed a density of 1.75 $\mathrm{g} / \mathrm{cm}^{3}(9,11,12,13)$. PEEK $(30 \%$ carbon fiber) material properties were derived from the literature and manufactures material data sheets ${ }^{(14,15)}$. Properties for titanium were taken from the material database in the simulation software. Material properties used in the analysis are given in Table 1 .

\begin{tabular}{|l|c|c|c|}
\hline \multicolumn{4}{|c|}{ Table 1: Material properties for FEA. } \\
\hline \multicolumn{1}{|c|}{ Material } & $\begin{array}{c}\text { Young's Modulus } \\
{[\mathrm{GPa}]}\end{array}$ & $\begin{array}{c}\text { Poisson's } \\
\text { Ratio }\end{array}$ & $\begin{array}{c}\text { Yield Strength } \\
{[\mathrm{MPa}]}\end{array}$ \\
\hline Ti (6Al, 4V) & 113.80 & 0.34 & 950.00 \\
PEEK (30\% C) & 20.00 & 0.42 & 131.00 \\
Cortical Bone & 11.64 & 0.40 & 114.00 \\
\hline
\end{tabular}

The stress analysis environment in the commercial analysis software Autodesk Inventor Professional $2013 \AA$ was used to perform linear, single-point, static FEM analyses on both constructs. This analysis uses at least $2^{\text {nd }}$ order solid tetrahedral elements and utilizes h-p adaptation refinements to converge to, in this analysis, the Von Mises stress. (Simply put, an $h$ adaptation refines the mesh in high-error areas to increase mesh accuracy and a $p$ adaptation refines the polynomial approximation used within each element. An h-p

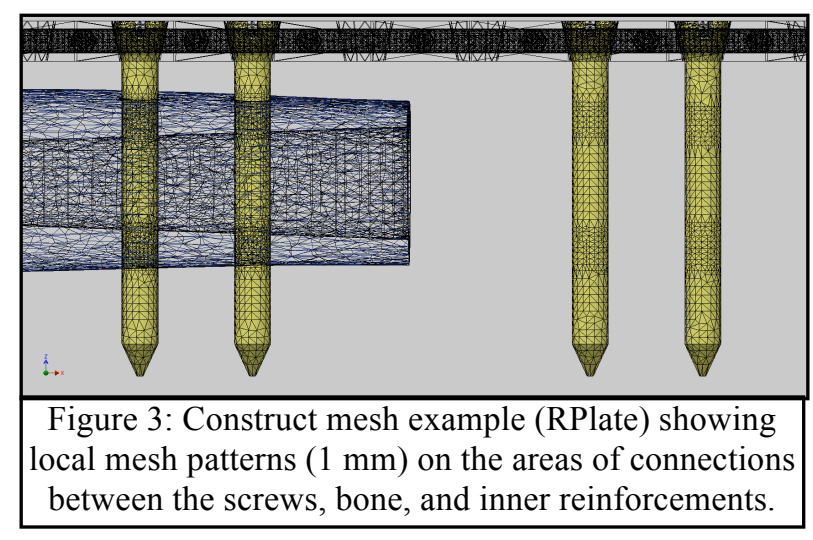


adaptation takes advantage of both methods and is considered the most efficient technique for dealing with errors found in FEM approximations.) The constructs averaged approximately 756,000 nodes, 482,000 elements and 500,000 nodes, 323,000 elements for the RPlate and SPlate respectively. An adaptive mesh refinement was set to converge on the Von Mises Stress with a stop criterion of $5 \%$, and a 0.75 h-refinement threshold. Localized mesh $1 \mathrm{~mm}$ in size was used in regions of interest, such as the screw-bone interface, throughout the constructs (Fig. 3).

\section{Results}

\section{1. $3 \mathrm{~mm}$ Gap Construct}

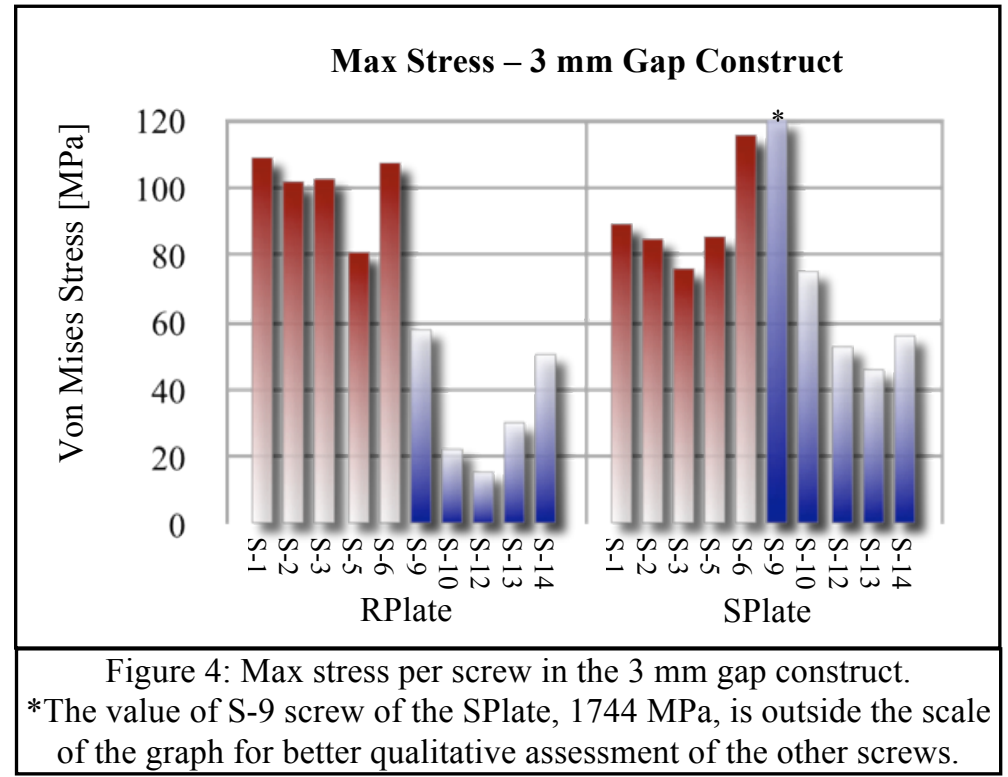

Figure 4 graphs the maximum stress per screw for the two constructs. The average stress per screw in the RPlate was lower than in the SPlate at $68 \mathrm{MPa}$ and $243 \mathrm{MPa}$, respectively (SPlate average $\sim 76 \mathrm{MPa}$ when discounting S-9). The maximum stress for the RPlate construct was $717 \mathrm{MPa}$ on the anterior end of the \#2 horizontal inner reinforcement (Fig. 5), and $1744 \mathrm{MPa}$ (To better visualize the relationship between the other screws, this value exceeds the scale of the graph in figure 4.) on the posterior side of the near cortex of S-9 for the SPlate construct (Fig. 6). A closer view of the high stress area and of the mesh refinement is shown in figure $7(\mathrm{a})(\mathrm{b})$. Maximum stress on the bone fragments was $31.8 \mathrm{MPa}$ proximally (S-5 near cortex), 31.2 MPa distally (S-9 near cortex) for the RPlate, and 40.2 MPa proximally (S-5 far cortex), 113.6 distally (S-9 near cortex) for the SPlate.

The screw strain for the two constructs ranged from 126 to $884 \mu$ strain in the RPlate and 372 to $14,270 \mu$ strain in the SPlate. Average strain per screw for the RPlate is $547 \mu$ strain and the average for the SPlate is $1,979 \mu$ strain. Maximum strain found in each construct was
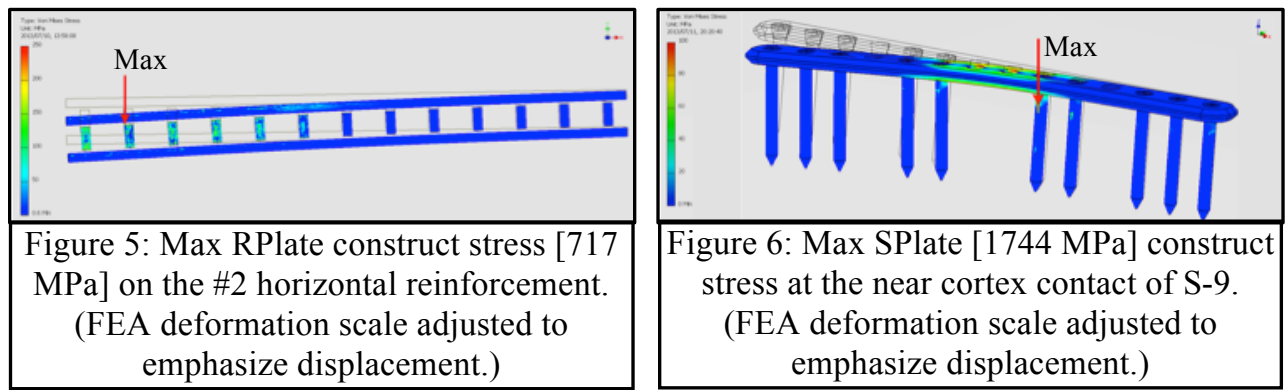
5,600 $\mu$ strain for the RPlate and 14,270 $\mu$ strain for the SPlate.

Maximum bone fragment displacement measured at the osteotomy for the RPlate construct approximately $0.47 \mathrm{~mm}$ for the proximal fragment and $0.17 \mathrm{~mm}$ for the distal fragment. Maximum fragment displacement for the SPlate construct within the osteotomy was approximately $0.37 \mathrm{~mm}$ proximally and $0.25 \mathrm{~mm}$ distally. Relative displacement between the bone fragments was $0.30 \mathrm{~mm}$ and $0.12 \mathrm{~mm}$ for the RPlate and SPlate respectively. The maximum displacement was $2.56 \mathrm{~mm}$ for the RPlate construct and $1.84 \mathrm{~mm}$ for the SPlate construct at the far proximal end of the tibia (intercondyloid eminence) for both constructs. Gap closure did not occur in either construct.

\subsection{Non-Gap Construct}

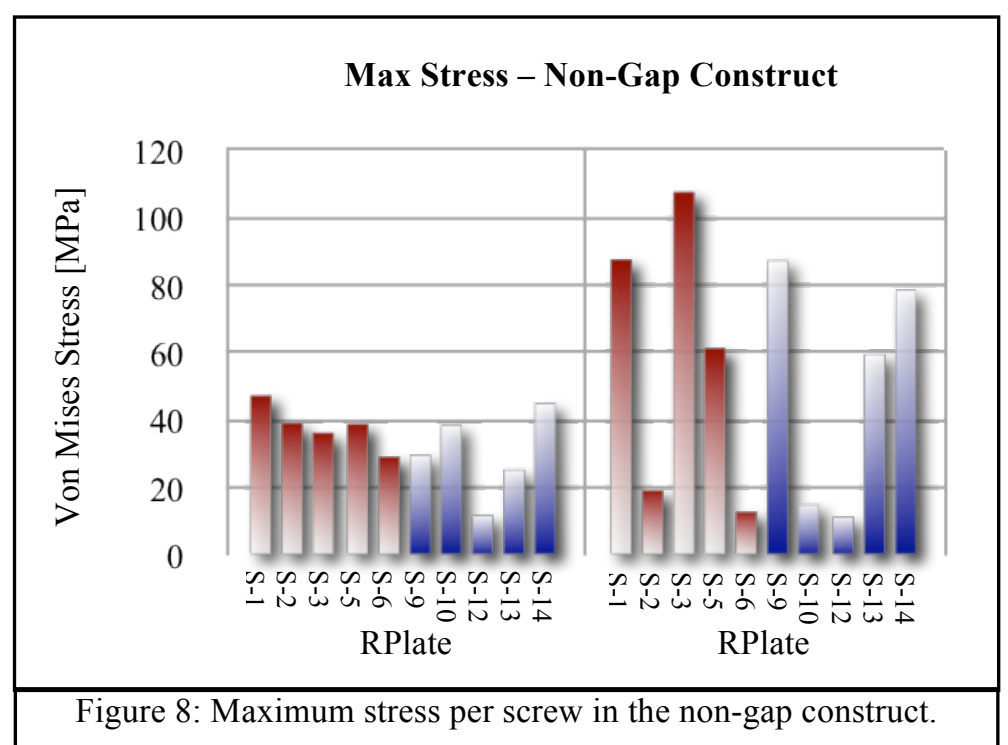

Maximum stress per screw in the non-gap construct is shown in figure 8 . The average screw stress for the RPlate construct was approximately 34.2 MPa and the SPlate construct averaged approximately 54.2 $\mathrm{MPa}$. The highest construct stresses were $803 \mathrm{MPa}$ in the RPlate construct on the posterior side of the $2^{\text {nd }}$ horizontal inner reinforcement (Fig. 9) and $209 \mathrm{MPa}$ on the posterior side of the S-1 plate hole for the SPlate (Fig. 10). The maximum stress found in the bone fragments of each construct were $10.8 \mathrm{MPa}$ proximally (S-6 near cortex), 9.0 MPa distally (S-13 near cortex) for the RPlate, and 10.0 MPa proximally (S-1 near cortex), 6.9 MPa distally (S-13 near cortex) for the SPlate. Stress within the osteotomy is higher when using the RPlate, 5.7 MPa proximally, 4.3 $\mathrm{MPa}$ distally compared with 2.0 MPa proximally, 1.9 MPa distally for the SPlate (Fig. 11). 

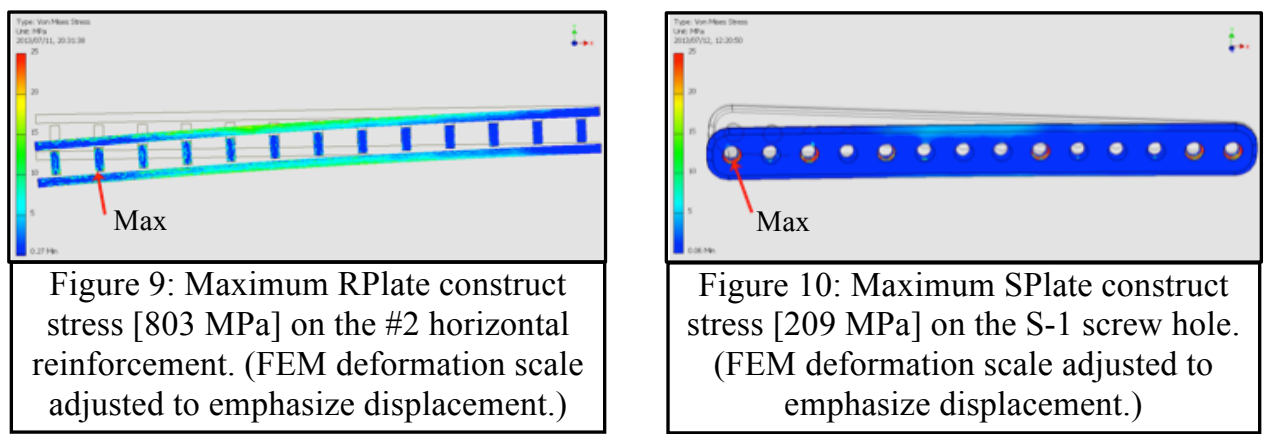

Screw strain ranged from 94 to $382 \mu$ strain in the RPlate and 91 to $896 \mu$ strain in the SPlate. The average strain per screw was 277 and $464 \mu$ strain for the RPlate and SPlate constructs respectively. The maximum strain found in the RPlate construct was 7,674 $\mu$ strain. For all other tests, the maximum strain was found at the same point as the maximum stress, however, for the SPlate construct with no fracture gap, the maximum, $988 \mu$ strain, was found at the S-3 plate hole rather than the S-1 plate hole where the maximum stress was located; the strain at that point was $896 \mu$ strain.

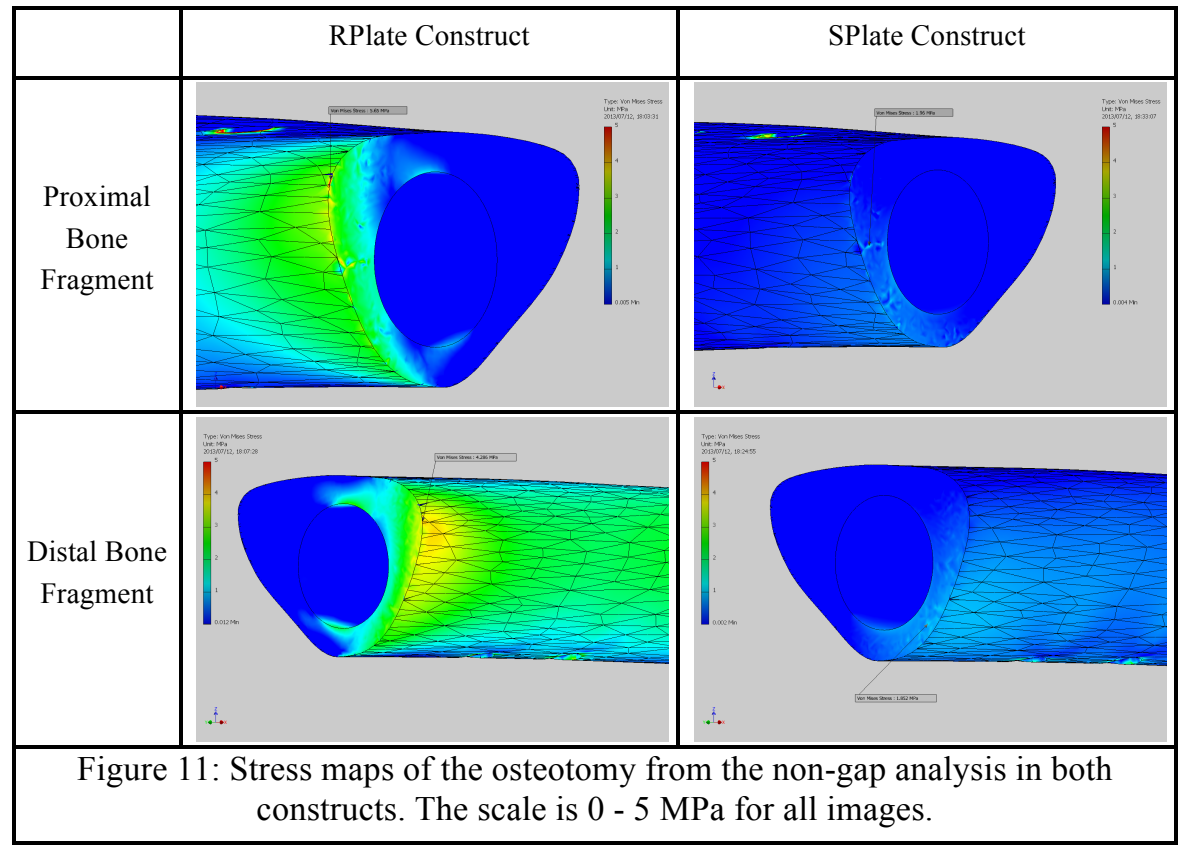

Maximum displacement for the constructs was $0.76 \mathrm{~mm}$ and $0.39 \mathrm{~mm}$ for the RPlate and SPlate respectively, and was located at the far proximal end of the tibia (intercondyloid eminence) for both constructs. The maximum relative displacement between the bone fragments at the osteotomy was very small $(<0.01 \mathrm{~mm})$ for both constructs.

\section{Discussion}

\subsection{3 mm Gap Construct}

In the RPlate construct, stress and strain of the screws on the proximal half of the fracture have slightly higher values than in the SPlate construct. However, the opposite appears to be true on the distal half of the fracture, even when the high value found on the S9 screw of the SPlate is excluded from the average. This effect is likely due to the complete fixation of the distal bone fragment, i.e., motion is allowed in the proximal bone fragment but not in the distal fragment. The high stress and strain on S-9 is not seen when using the 
RPlate construct, and those high values are well over the yield point of the titanium screw, which could lead to screw breakage and even construct failure. The cause of the high stress and strain on S-9 appears to be caused by a combination of geometry, mesh and loading conditions. The region of interest is at the approximate point where the near cortex of the distal bone fragment meets the screw. The high stress/strain values persist throughout several mesh refinements (Fig. 7). These high values occur on the smooth surface of the screw, and not on a corner or sharp feature, therefore appearing to be a weak point in the SPlate construct when loaded in a manner that produces a moment of rotation in the system.

The stress on the proximal bone fragment was very similar between the two constructs. However, on the distal bone fragment, the highest stress on the SPlate construct was nearly triple that of the RPlate construct, 114 versus $40 \mathrm{MPa}$. It has reached the yield strength of cortical bone (114 $\mathrm{MPa}$ in this analysis) and could possibly lead to peri-implant fracture, again, at the S-9 position.

One issue to consider with the RPlate is the maximum stress found on the inner reinforcement, which could indicate a problem with the reinforcement cutting out of the PEEK outer shell. However, this problem may be corrected with a simple design change rounding off or adding a fillet to the ends of the inner reinforcements.

Claes et al. ${ }^{(16)}$ determined that fracture stability is increased in the later healing stages when initial interfragmentary motion is relatively high, between 0.1 and $2.7 \mathrm{~mm}$. In this construct, the overall displacement for the RPlate was $0.3 \mathrm{~mm}$ and the SPlate was $0.12 \mathrm{~mm}$ indicating that the RPlate construct has a greater potential for fracture stability in later healing. However, it is unclear how the construct will function after sufficient secondary healing occurs since fracture callus gained over time was not a part of this analysis. Furthermore, gap closure did not occur in neither the RPlate nor the SPlate analysis so other interfragmentary conditions, apart from bone fragment displacement, are unknown.

\subsection{Non-Gap Construct}

The average stress and strain per screw in the non-gap construct shows a decrease when comparing the RPlate to the SPlate, which may indicate less of a chance of screw breakage with the RPlate. The proximal screw stress and strain average values are slightly higher than the average distal values for both constructs. While the highest stresses on the proximal and distal bone fragments are similar between the two constructs, the stress within the osteotomy is higher for the RPlate construct compared with the SPlate construct. Figure 8 shows how the SPlate construct is causing stress shielding within the fracture gap whereas the RPlate construct allows more stress generation. According to the bone mechanostat hypothesis, the amount of strain within the osteotomy of the RPlate construct is approximately at the lower threshold for minimally effective bone remodeling strain, but the SPlate construct is only within the range for disuse-mode bone remodeling ${ }^{(17)}$.

\subsection{RPlate versus SPlate}

Titanium reinforced PEEK fracture plates are shown to outperform standard titanium plates in early fracture healing. When a gap between bone fragments is unavoidable, the RPlate allows crucial micro-motion, which is important for secondary bone healing. In a non-gap condition, the RPlate reduces stress shielding within the fracture gap when compared with the SPlate. In both gap and non-gap constructs, the RPlate reduces overall stress on the screws, which reduces the possibility of screw breakage. 


\subsection{Study Limitations}

This study contains several limitations. First, there is a lack of cancellous bone in the analysis. Typically, the ends of long bones are made of cancellous bone covered by cortical bone. This study assumes the tibia to be made only of cortical bone, which could affect load distribution producing differences in resultant stress and strain. However, the region of interest in this study, the tibial diaphysis, is made only of cortical bone so the effect is likely minimal. Second, muscle attachments were not simulated. The muscle attachments to the tibia involve some relatively strong muscles and would provide additional forces that are not accounted for here. The forces simulated in this study are meant to approximate what might be found in the first few days of recovery where a patient would not be able to fully stand on the leg but may rest the foot on the floor while otherwise supported with the assistance of crutches or a walker. Third, an experimental study to validate the results has not been performed and at the time of this writing plans have not yet been made for such a study.

\section{Conclusion}

This computational analysis shows that a titanium-reinforced PEEK fracture plate is superior to a standard titanium orthopedic plate in the initial stages of fracture healing. The RPlate displays an overall decrease in screw stress, consistency of the screw stress values across the length of the plate in a non gap construct, evidence of stress shielding reduction within the fracture gap, and the potential for a more stable fracture in later stages of healing when compared to the SPlate. Even without a mechanical analysis for validation, the computational analysis performed here is highly compelling, and is persuasive evidence for a deeper investigation of fracture plating of this type.

\section{References}

1. K. A. Egol, E. N. Kubiak, E. Fulkerson, F. J. Kummer, and K. J. Koval. Biomechanics of locked plates and screws. Journal of orthopaedic trauma, 18(8):488-493, 2004.

2. P. Comte and F. Straumann. Influence of unoccupied holes on the fatigue behaviour of bone fixation plates. In Biomechanics: Current Interdisciplinary Research, pages 459-464. Springer, 1985.

3. S. R. Davenport, R. W. Lindsey, R. Leggon, T. Miclau, and M. Panjabi. Dynamic compression plate fixation: a biomechanical comparison of unicortical vs bicortical distal screw fixation. Journal of orthopaedic trauma, 2(2):150, 1988.

4. K. Stoffel, U. Dieter, G. Stachowiak, A. Gachter, and M. S. Kuster. Biomechanical testing of the lcp - how can stability in locked internal fixators be controlled? Injury, 34, Supplement 2(0): $11-19,2003$.

5. S. M. Kurtz and J. N. Devine. Peek biomaterials in trauma, orthopedic, and spinal implants. Biomaterials, 28(32):4845-69, Nov 2007.

6. R. Huiskes and D. Nunamaker. Local stresses and bone adaption around orthopedic implants. Calcif Tissue Int, 36 Suppl 1:S110-7, 1984.

7. J. Sanderson, Y. Nakanishi, N. Murayama, T. Nishimura, T. Ishi, H. Mizuta, and H. Higaki. Polyetheretherketone in orthopedic plate design to facilitate fracture recovery. Journal of Biomechanical Science and Engineering, 6(2):126-133, 2011.

8. VAKHUM Public Dataset, Van Sint, Jan S., April 25, 2003, Department of Anatomy of the University of Brussels, October $2012<\mathrm{https}$ //www.ulb.ac.be/project/vakhum/index.html $>$.

9. L. Peng, J. Bai, X. Zeng, and Y. Zhou. Comparison of isotropic and orthotropic material property assignments on femoral finite element models under two loading conditions. Medical engineering \&amp; physics, 28(3):227-233, 2006.

10. D. Kettelkamp, D. Wenger, E. Chao, C. Thompson, et al. Results of proximal tibial osteotomy. the effects of tibiofemoral angle, stance-phase flexion-extension, and medialplateau force. The Journal of bone and joint surgery. American volume, 58(7):952-960, 
1976.

11. J. Y. Rho, L. Kuhn-Spearing, and P. Zioupos. Mechanical properties and the hierarchical structure of bone. Medical engineering \&amp; physics, 20(2):92-102, 1998.

12. D. C. Wirtz, N. Schiffers, T. Pandorf, K. Radermacher, D. Weichert, and R. Forst. Critical evaluation of known bone material properties to realize anisotropic fe-simulation of the proximal femur. J Biomech, 33(10):1325-30, Oct 2000.

13. D. T. REILLY and A. H. BURSTEIN. The mechanical properties of cortical bone. The Journal of Bone and Joint Surgery, 56(5):1001-1022, 1974.

14. S. M. Kurtz and J. N. Devine. Peek biomaterials in trauma, orthopedic, and spinal implants. Biomaterials, 28(32):4845-69, Nov 2007.

15. Z. Inc. Peek vs metal: Why plastic is better. Technical whitepaper, Zeus Industrial Products, 3737 Industrial Blvd. Orangeburg, SC 29118 United States, 2005.

16. L. Claes, P. Augat, G. Suger, and H. J. Wilke. Influence of size and stability of the osteotomy gap on the success of fracture healing. J Orthop Res, 15(4):577-84, Jul 1997.

17. H. M. Frost. Bone's mechanostat: a 2003 update. The Anatomical Record Part A: Discoveries in Molecular, Cellular, and Evolutionary Biology, 275(2):1081-1101, 2003. 